\title{
Papers
}

\section{The psychosocial impact of bilateral prophylactic mastectomy: prospective study using questionnaires and semistructured interviews}

\author{
Mal Bebbington Hatcher, Lesley Fallowfield, Roger A'Hern
}

\begin{abstract}
Objectives To investigate the psychosocial impact of bilateral prophylactic mastectomy for women with increased risk of breast cancer and to identify, preoperatively, risk factors for postoperative distress. Design Prospective study using interviews and questionnaire assessments.

Setting Participants' homes throughout the United Kingdom.

Participants 143 women with increased risk of developing breast cancer who were offered bilateral prophylactic mastectomy and who accepted or declined the surgery; a further 11 were offered surgery but deferred making a decision.

Main outcome measures Psychological and sexual morbidity.

Results Psychological morbidity decreased

significantly over time for the 79 women who chose to have surgery (accepters): 58\% (41/71) preoperatively $v 41 \%(29 / 71) 6$ months postoperatively (difference in percentages $17 \%, 95 \%$ confidence interval $2 \%$ to $32 \%$; $\mathrm{P}=0.04)$ and $60 \%(39 / 65)$ preoperatively $v 29 \%$ (19/65) 18 months postoperatively (31\%, 15\% to $47 \%$; $\mathrm{P}<0.001)$. Psychological morbidity in the 64 women who declined surgery (decliners) did not decrease significantly: $57 \%(31 / 54)$ at baseline $v 43 \%(23 / 54)$ at 6 months $(14 \%, 0 \%$ to $29 \% ; \mathrm{P}=0.08)$ and $57 \%$ $(29 / 52)$ at baseline $v 41 \%(21 / 52)$ at 18 months $(16 \%$; $-2 \%$ to $33 \% ; \mathrm{P}=0.11)$. Greater than normal proneness to anxiety was more common in the decliners than in the accepters: $78 \%(45 / 58) v 56 \%$ $(41 / 73)(22 \%, 6 \%$ to $38 \% ; \mathrm{P}=0.006)$. Accepters were more likely than decliners to believe it inevitable that they would develop breast cancer (32\% (24/74) v 10\% $(6 / 58)$ (difference in percentages $22 \%, 9 \%$ to $35 \%$; $\mathrm{P}=0.003)$ ), and decliners were more likely to believe that screening could help (92\% (55/60) v 74\% (55/74) $(18 \%, 5 \%$ to $31 \% ; \mathrm{P}=0.007))$. Level of sexual discomfort and degree of sexual pleasure did not change significantly over time in either of the two groups.

Conclusions Bilateral prophylactic mastectomy may provide psychological benefits in women with a high risk of developing breast cancer.
\end{abstract}

\section{Introduction}

Bilateral prophylactic mastectomy may significantly reduce development of breast cancer in women at increased risk, ${ }^{1}$ but as it is a radical surgical intervention the psychological costs and benefits are important to establish. Published reports tend to be personal accounts of the decision to undergo surgery or of living with the threat of the disease, ${ }^{23}$ or they are case studies describing women's reactions to the impact of the disease on themselves and their family and the difficulties they faced in decision making. ${ }^{4}$ One study reported that women's decisions to have prophylactic mastectomy were based on their biopsy history, subjective perceptions of risk, and degree of worry about cancer. ${ }^{6}$

Postoperative regret about having decided to undergo surgery was reported in a retrospective study. The proportion of women expressing regret was small ( $6 \%$ of 370 participants), and regret was more common in those where discussion about the procedure was initiated by a physician, not the patient. Ninety per cent of the women who regretted surgery had not received preoperative counselling. Positive outcomes after bilateral prophylactic mastectomy were reported in another retrospective study, including favourable psychological and social outcomes and decreased emotional concern about developing the disease. ${ }^{8}$ To date, little prospective research has been published in large samples of women about the factors influencing decision making or the psychosocial implications of prophylactic surgery.

Important psychological benefits from surgery may include a reduction in chronic anxiety and worry, in distress associated with false positive mammography results, and in dependence on screening and self examination. ${ }^{9}$ For women who fear developing the disease, the putative psychological benefits of surgery may well outweigh any negative consequences; none the less, it is important to consider the psychological costs and to examine the long term impact on women who are offered the procedure but decline it.

We measured-by using interviews and questionnaire assessments-psychological morbidity in women at increased risk of developing breast cancer who were offered bilateral prophylactic mastectomy, regardless of whether they accepted the option.

\author{
Cancer Research \\ Campaign \\ Psychosocial \\ Oncology Group, \\ Bland Sutton \\ Institute, Royal Free \\ and University \\ College Medical \\ School, London \\ W1P 7PL \\ Mal Bebbington \\ Hatcher \\ research psychologist \\ Lesley Fallowfield \\ professor of \\ psycho-oncology \\ Mulberry House, \\ Royal Marsden \\ NHS Trust, London \\ SW3 6JJ \\ Roger A'Hern \\ statistician \\ Correspondence to: \\ L Fallowfield \\ l.fallowfield@ucl.ac.uk \\ BMJ 2001;322:1-7
}




\section{Method}

\section{Participants}

Women at increased risk of developing breast cancer were referred to us by clinicians (20 surgeons, 4 geneticists, 4 medical oncologists, and 1 psychiatrist) working in 20 participating centres throughout the United Kingdom. Most referrals (54\%) came from the North West health region. All women gave written informed consent to join the study, which had local and regional ethics approval. Interviews and completion of questionnaires were conducted in the participants' homes.

Eligibility criteria were having a family history of breast cancer or having sufficiently high risk factors for bilateral prophylactic mastectomy to be offered. Genetic status was determined by the referring clinicians. We did not know the risk level used by the referring clinicians to select patients to be offered surgery. Women reported being informed of their risk either of carrying the gene or of developing the disease. Most women in both groups (57\% (42/74) of those who chose to have surgery (accepters), 71\% (41/ 58 ) of those who declined surgery (decliners); non-significant difference) reported a risk of carrying the gene of between 1 in 2 and 1 in 4 .

Of 168 women eligible to join the study, 7 declined and 4 consented (but contact was lost with these 11 before they completed any assessments); a further 2 declined follow up assessments; and another was lost to follow up after first interview. Of the 154 women recruited to the study, 79 chose surgery, 64 declined, and 11 deferred making a decision while awaiting results of gene testing, completing their family, or seeking further information. These 11 were not included in any analysis.

Most women (73\% (58/79) of accepters, 83\% $(52 / 63)$ of decliners) were in paid occupations. The age range was similar for both groups (26-57 (median 38) years $v$ 22-56 (40) years). Most women had children $(81 \%(64 / 79)$ v $75 \%(48 / 64))$.

Women referred from specialist centres had had access to geneticists (who conducted genetic assessment and counselling) and to clinical psychologists or psychiatrists for psychological counselling. Most clinicians who referred women to the study, however, were surgeons who had themselves assessed the risk and provided risk counselling. Few of the women seen by surgeons had been offered psychological counselling. The women referred by geneticists had often not seen a surgeon at the time of first interview and did not all have sufficient information about the surgery.

Ten women chose not to have reconstruction after surgery. Sixty four had implants, two had tranverse rectus abdominus muscle flaps, and one woman had a latissimus dorsi flap on a breast that had previously been operated on for cancer while an implant was placed in the other breast. Details of postoperative complications will not be known until a qualitative analysis of all audiotaped interviews is complete. Decliners were offered regular surveillance (mammography and clinical examination).

\section{Interviews}

All women had their first interview as soon as possible after they were referred to the study. The accepters were interviewed again at 6 and 18 months postopera- tively; the mean time from first interview until surgery was 24 weeks (median 17 weeks, range 2 days to 125 weeks). Women who declined or deferred making a decision were interviewed again 18 months after the first interview. The interviews were semistructured, with questions phrased to elicit information on decision making, perceptions of risk, and psychosocial implications of surgery. Content analysis of transcribed tapes is continuing and will be reported elsewhere.

Most women made their decision on whether to have surgery at the time of the first interview, regardless of whether they had seen a surgeon or felt they had all the necessary information. A small minority changed their decision at a later date, on receipt of a positive or negative blood test result.

\section{Questionnaires}

Six questionnaires were used in the study.

- The "general health questionnaire 30" was developed for use as a screening tool to determine psychiatric morbidity in clinical or community settings. ${ }^{10}$ It has excellent reliability and has been successfully validated against the clinical interview schedule. It is a self administered, 30 item questionnaire (with each item rated on a 4 point scale).

- The "Spielberger state-trait anxiety inventory" consists of two self administered, 20 item questionnaires, with each item rated on a 4 point scale. ${ }^{11}$ It is a well researched clinical tool for evaluating current state of anxiety (state) and proneness to anxiety (trait) and has been successfully used in previous work with women with breast cancer. ${ }^{12}$

- The "sexual activity questionnaire" was developed to assess sexual functioning in the Tamoplac and IBIS chemoprevention trials. ${ }^{13} 14$ The questionnaire has test/retest reliability, and normative data from over 100 women aged between 30 and 65 from the general population are available. The questionnaire comprises three sections (hormonal status, reasons for sexual inactivity, and sexual functioning) and has been factor analysed into three scales (pleasure, discomfort, and habit). It is currently being used in many other trials of cancer therapy and seems acceptable to most women to whom it has been administered, including patients with advanced ovarian cancer. ${ }^{15}$

- The "ways of coping questionnaire" (revised) is a 66 item checklist devised to assess coping strategies used to deal with a specific stressful event. ${ }^{16}$ The questionnaire items are rated on a 4 point, Likert-type scale. The questionnaire has been factor analysed to predict eight scales of coping strategies: problem focused; detachment; wishful thinking; seeking social support; focusing on the positive; self blame; tension reduction; and keeping to self.

- The "risk perception questionnaire" contains five questions that assess knowledge of risk. ${ }^{17}$ Analysis consists of comparing risk estimates reported by women with objective risk estimates and looking at the frequency of risk values reported. This questionnaire was used in a study examining the psychological support needs of women at increased genetic risk of

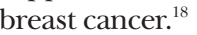

- The "body image scale" is used to assess women's perception of their body image, applicable across disease sites and treatment methods. ${ }^{19}$ The questionnaire has 10 items scored on a 4 point, Likert-type 
Table 1 Comparisons of percentages of women scoring $\geqslant 4$ (threshold for possible psychological morbidity) on general health questionnaire, based on numbers of women completing questionnaires at all relevant time points

\begin{tabular}{|c|c|c|c|c|c|c|}
\hline \multirow[b]{3}{*}{ Assessments } & \multicolumn{3}{|c|}{ Accepters* } & \multicolumn{3}{|c|}{ Decliners† } \\
\hline & & Difference (\%) & & & Difference (\%) & \\
\hline & $\%$ (proportion) & $(95 \% \mathrm{CI})$ & $P$ value & $\%$ (proportion) & $(95 \% \mathrm{CI})$ & $P$ value \\
\hline Baseline $v 6$ months & $58(41 / 71)$ v $41(29 / 71)$ & 17 (2 to 32$)$ & 0.04 & $57(31 / 54)$ v $43(23 / 54)$ & $14(0$ to 29$)$ & 0.08 \\
\hline Baseline $v 18$ months & $60(39 / 65)$ v $29(19 / 65)$ & 31 (15 to 47 ) & $<0.001$ & $57(29 / 51)$ v $41(21 / 51)$ & 16 (-2 to 33$)$ & 0.11 \\
\hline
\end{tabular}

scale. The scale has been subjected to extensive psychometric testing in the United Kingdom with data provided by several centres.

All the questionnaires were administered to all women at the first interview. At 6 months and 18 months after their first interview, the decliners completed the general health questionnaire, the Spielberger (state) questionnaire, and the sexual activity questionnaire. At 6 months and 18 months postoperatively, the accepters completed these same three questionnaires, plus the body image scale.

\section{Analysis}

As the distributions of the outcome measures were frequently skewed, non-parametric statistical tests were used. The $\chi^{2}$ test was used to compare differences in proportions, except where the observations were paired, in which case the McNemar test was used. Paired, continuous observations were compared by using the Wilcoxon test, and independent groups were compared by using the Mann-Whitney U test.

Baseline statistical analysis included all women who completed the assessment at the first interview. In subsequent analyses, only those women who completed assessments at each time point were included.

Ten women did not have a third interview; of these, five had had their operation too late in the study to have an 18 month postoperative interview, three had moved too far away to make it financially feasible to attend, and two were lost to follow up (despite intensive efforts to trace them). These two women were lost to follow up at a late stage and had therefore been included in the earlier analysis.

\section{Results}

General health questionnaire

Table 1 shows the results from the general health questionnaire. The scores were compared over time within and between the two groups. The proportion of accepters scoring $\geqslant 4$ (the threshold for possible psychological morbidity, using the "GHQ" scoring system $^{10}$ ) reduced over time, both between the preoperative (baseline) and the 6 month postoperative assessment and between the preoperative and the 18 month postoperative assessment. Psychological morbidity decreased significantly over time among the accepters, and the longer the time from surgery, the greater the decrease. The proportion of decliners scoring $\geqslant 4$ did not differ significantly between the first (baseline) and the 6 month assessment. Over 50\% of decliners had psychological morbidity at the first assessment, and this did not decrease significantly over an 18 month period. No significant differences existed between the two groups of women at any of the three time points.

\section{Spielberger state-trait anxiety inventory}

The women's scores for anxiety as a trait (tested at baseline) were compared with published normative values for women in this age group; a significantly higher proportion of decliners than accepters were prone to anxiety (table 2).

The women's scores for anxiety at the time of assessment (state anxiety) were compared with published normative values for women in this age group. Table 3 shows the results. The proportion of accepters with anxiety above the normative score decreased between the preoperative and the 6 month postoperative assessment and between the preoperative and the 18 month postoperative assessment. Although anxiety levels were high among the accepters, these decreased significantly over time, with the decrease being greater the longer the time from surgery (table 4). The proportion of decliners scoring above the normative value did not differ significantly between the baseline and the 6 month assessment and the baseline and the 18 month assessment. Over 50\% of decliners were anxious, and their anxiety levels remained high 18 months after the first assessment. No significant differences in anxiety score existed between the two groups of women at any of the three time points.

\section{Sexual activity questionnaire}

Table 5 shows the results of the sexual activity questionnaire. Sexual discomfort changed little over time within or between groups, with median scores being very close to the maximum of 6 (indicating no discomfort). Among accepters, the median was 6 at all times. Among decliners the median score did not change significantly over the three time points. No significant differences in sexual pleasure were found between or within groups. Degree of sexual pleasure did not change significantly over time in either of the two groups.

\section{Ways of coping questionnaire}

Table 6 shows the results of the ways of coping questionnaire. The median score for using problem

Table 2 Comparisons of percentages of women scoring above normative score of 35.6 (indicating anxiety proneness) on Spielberger trait anxiety questionnaire, and mean scores

\begin{tabular}{lcccc} 
& Surgery $(\mathbf{n}=\mathbf{7 3})$ & Decliners $(\mathbf{n}=\mathbf{5 8})$ & $\begin{array}{c}\text { Difference }(\mathbf{\%}) \\
(\mathbf{9 5 \%} \mathbf{C l})\end{array}$ & $\mathbf{P}$ value \\
\hline$\%($ No) of women & $56(41)$ & $78(45)$ & $22(6$ to 38$)$ & 0.006 \\
\hline Mean score (range) & $38(35-42)$ & $42(37-49)$ & $2(-2$ to 6$)$ & 0.36 \\
\hline
\end{tabular}

The higher the score, the greater the proneness to anxiety. 
Table 3 Comparisons of percentages of women scoring above normative score of 36.2 (indicating high anxiety) on Spielberger state anxiety questionnaire, based on numbers of women completing questionnaires at all relevant time points

\begin{tabular}{|c|c|c|c|c|c|c|}
\hline \multirow[b]{2}{*}{ Assessments } & \multicolumn{3}{|c|}{ Accepters } & \multicolumn{3}{|c|}{ Decliners } \\
\hline & $\%$ (proportion) & $\begin{array}{l}\text { Difference (\%) } \\
(95 \% \mathrm{CI})\end{array}$ & $P$ value & $\%$ (proportion) & $\begin{array}{c}\text { Difference (\%) } \\
(95 \% \mathrm{CI})\end{array}$ & $P$ value \\
\hline Baseline $v 6$ months & $69(47 / 68)$ v $40(27 / 68)$ & $29(15$ to 44$)$ & $<0.001$ & $54(26 / 48)$ v 54 (26/48) & $0(-13$ to 13$)$ & 1.00 \\
\hline Baseline $v 18$ months & $71(45 / 63)$ v $41(26 / 63)$ & 30 (14 to 46$)$ & 0.001 & $52(24 / 46)$ v $50(23 / 46)$ & $2(-11$ to 15$)$ & 1.00 \\
\hline
\end{tabular}

The higher the score, the greater the anxiety.

Table 4 Comparisons of women's raw scores on Spielberger state anxiety questionnaire, based on numbers of women completing questionnaires at all relevant time points

\begin{tabular}{|c|c|c|c|c|c|c|}
\hline \multirow[b]{2}{*}{ Assessments } & \multicolumn{3}{|c|}{ Accepters } & \multicolumn{3}{|c|}{ Decliners } \\
\hline & Median score & $\begin{array}{l}\text { Median difference } \\
\quad(95 \% \mathrm{CI})\end{array}$ & $P$ value & Median score & Median difference $(95 \% \mathrm{Cl})$ & $P$ value \\
\hline Baseline $v 6$ months & $45.3 \vee 32$ & $4(2.2$ to 10$)$ & $<0.001$ & $38.5 \vee 37$ & 0.5 (-3 to 4$)$ & 0.44 \\
\hline Baseline $v 18$ months & 43.5 v 33 & $6(2$ to 10$)$ & $<0.001$ & $38.5 \vee 36$ & 1 (-3 to 2$)$ & 0.27 \\
\hline
\end{tabular}

The higher the score, the greater the anxiety.

Table 5 Median scores for sexual discomfort ${ }^{\star}$ and sexual pleasure† over time

\begin{tabular}{|c|c|c|c|c|c|c|}
\hline \multirow[b]{3}{*}{ Assessments } & \multicolumn{3}{|c|}{ Accepters ( $n=45)$} & \multicolumn{3}{|c|}{ Decliners ( $n=34$ ) } \\
\hline & \multicolumn{3}{|c|}{ Median difference $\mathbf{( 9 5 \%}$} & \multicolumn{3}{|c|}{ Median difference } \\
\hline & Score & Cl) & $P$ value & Score & $(95 \% \mathrm{CI})$ & $P$ value \\
\hline \multicolumn{7}{|l|}{ Sexual discomfort: } \\
\hline Baseline $v 6$ months & $6 v 6$ & $0(0$ to 0$)$ & 0.93 & $5 \vee 6$ & $0(0$ to 0$)$ & 0.75 \\
\hline Baseline $v 18$ months & $6 v 6$ & $0(0$ to 0$)$ & 0.56 & $5 v 5.5$ & $0(0$ to 0$)$ & 0.41 \\
\hline \multicolumn{7}{|l|}{ Sexual pleasure: } \\
\hline Baseline $v 6$ months & $13.5 \vee 14$ & 0 (-1 to 2$)$ & 0.44 & $12.5 \vee 15$ & $0(-1$ to 0$)$ & 0.50 \\
\hline Baseline $v 18$ months & $13.5 \vee 14$ & $0(-1$ to 1$)$ & 0.69 & $13.5 \vee 13.5$ & $0(-1$ to 0$)$ & 0.65 \\
\hline
\end{tabular}

* $S$ cale of 0 to 6 ( $6=$ no discomfort). $†$ Scale of 0 to 18 (18=high sexual pleasure).

focused coping was significantly higher among accepters than among decliners; the median score for using detachment as a coping mechanism was significantly higher among decliners than among accepters.

Table 6 Median scores* for problem focused and detachment coping styles, according to results of ways of coping questionnaire

\begin{tabular}{lcccc} 
Coping style & Accepters $(\mathbf{n}=\mathbf{7 5})$ & Decliners $(\mathbf{n}=\mathbf{5 9})$ & $\begin{array}{c}\text { Median difference } \\
\mathbf{( 9 5 \%} \mathbf{C I})\end{array}$ & P value \\
\hline Problem focused & 16 & 14 & $3(0$ to 5$)$ & 0.03 \\
\hline Detachment & 3 & 7 & $3(2$ to 5$)$ & $<0.001$ \\
\hline
\end{tabular}

${ }^{*} \mathrm{~A}$ higher score indicates more use of the coping style.

Table 7 Women's personal risk estimates from risk perception questionnaire: percentage frequency of answers to question, "What do you think is your lifetime risk of developing breast cancer?"

\begin{tabular}{lcc} 
& \multicolumn{2}{c}{ Percentage frequency } \\
\cline { 2 - 3 } Risk estimate & Accepters $(\mathbf{n}=\mathbf{7 4})$ & Decliners $(\mathbf{n}=\mathbf{5 7})$ \\
\hline Inevitable & 32 & 10 \\
\hline $80 \%$ & 3 & 0 \\
\hline 1 in 2 & 27 & 19 \\
\hline 1 in 3 & 20 & 35 \\
\hline 1 in 4 & 10 & 17 \\
\hline 1 in 5 & 1 & 2 \\
\hline 1 in 6 & 1 & 2 \\
\hline 1 in 8 & 0 & 2 \\
\hline 1 in 10 & 3 & 4 \\
\hline 1 in 12 & 0 & 0 \\
\hline 1 in 20 & 1 & 5 \\
\hline 1 in 50 & 0 & 0 \\
\hline 1 in 100 & 1 & 2 \\
\hline
\end{tabular}

Comparison of inevitable $v$ not inevitable, $\mathrm{P}=0.001$.

Mann-Whitney test for trend over all categories, $\mathrm{P}<0.005$

\section{Body image scale}

When the body image questionnaire was administered postoperatively to the accepters (most of whom had had immediate reconstruction) at the 6 and 18 month interviews, no differences in the median score of 4 (range 0-30, with 0 indicating most positive body image) were detected (median change 0, 95\% confidence interval 0 to $1 ; \mathrm{P}=0.84)$. Scores were similar to those published recently of women with breast cancer who had immediate reconstruction. ${ }^{20}$

\section{Risk perception questionnaire}

Table 7 shows the women's perceptions of personal risk from breast cancer. Although most women in both groups reported perceived risk levels of between 1 in 2 and 1 in 4, the accepters overall tended to report higher lifetime risks of developing breast cancer than the decliners. In particular, accepters were more likely than decliners to believe it inevitable that they would develop the disease. Decliners were more likely than accepters to believe that screening could help (92\% $(55 / 60)$ v 74\% (55/74); difference in percentages $18 \%$ (95\% confidence interval $5 \%$ to $31 \%) ; \mathrm{P}=0.007)$.

\section{Investigatory and genetic tests}

Accepters were more likely than decliners to have had an investigatory test (fine needle aspiration, biopsy, or lumpectomy) (43\% (34/79) v 19\% (12/64); difference in percentages $24 \%(10 \%$ to $39 \%) ; \mathrm{P}=0.002)$ or a gene test $(29 \%(23 / 79)$ v $5 \%(3 / 64)$; difference $24 \%$ (13\% to $36 \%) ; \mathrm{P}<0.001)$. 


\section{Discussion}

Our primary aim was to evaluate the psychosocial impact of bilateral prophylactic mastectomy by comparing psychological morbidity in women who have had the procedure with women who have been offered but declined the procedure. High levels of psychological morbidity and anxiety before surgery reduced significantly over time after surgery. In women who declined surgery and opted for regular surveillance and screening, high levels of psychological morbidity and anxiety were maintained.

Our results are concordant with those from a US study which hypothesised that bilateral prophylactic mastectomy reduced chronic anxiety and worry, while women attending a family history clinic but not participating in any other prevention programme had increased anxiety. ${ }^{9}$ Conversely, other authors reported that 24 women with a family history who did not attend a family history clinic had significantly higher anxiety scores than 47 women with a family history who were attending a clinic and participating in a chemoprevention trial comparing tamoxifen with placebo. ${ }^{21}$ These authors surmised that participation in a prevention programme, or attendance at a specialist clinic, alleviated anxiety.

In our study the women who declined surgery had significantly higher scores for anxiety as a personality trait than those who had surgery, which may explain why the high levels of anxiety and psychological morbidity among decliners did not decrease over time. The women who declined also tended to use detachment to cope, rather than the problem focused approach used more frequently by those who had surgery. The statements reflecting use of detachment (such as "I try to forget the whole thing") are much more passive than the problem focused statements (such as "I'm making a plan of action and following it"). Although they seemed to be more anxious, the women who declined surgery were less inclined to act on their anxiety.

It is encouraging to note that the women who had surgery (most of whom had had immediate reconstruction) maintained a positive body image and reported few or no changes in sexual activity at each time point, although longer follow up studies are needed.

Further research is also needed to look at the best method of ensuring that risk perception is accurate. A recent study found that genetic counselling produced only a modest shift in the accuracy of perceived lifetime risk. ${ }^{22}$ We found that $32 \%$ of women who had surgery believed it inevitable that they would develop breast cancer. If women are making decisions based on inaccurate perceptions they might regret these decisions later. Although our study found no evidence for this up to 18 months after surgery, future research needs to include a longer follow up. Inaccurate perceptions of lifetime risk, coupled with the greater number of investigatory tests, confirms another report that women choosing surgery had undergone more biopsies and reported higher risk estimates than those who declined. ${ }^{6}$ In another study of women at high genetic risk, entry into a chemoprevention trial was higher in women who believed themselves to be at greater personal risk. ${ }^{23}$
What is already known on this topic

Little is known of the psychological and social impact of bilateral prophylactic mastectomy for women with increased risk of developing breast cancer

Women's decisions to undergo the surgery may be based on biopsy history, subjective perceptions of risk, and degree of worry about cancer

\section{What this study adds}

Bilateral prophylactic mastectomy reduces psychological morbidity and anxiety and does not have a detrimental impact on women's body image or sexual functioning

Women who choose such surgery have had more investigative tests than women who decline; they also have a higher, often inaccurate, perception of their risk of developing breast cancer

Genetic counsellors need to ensure that women's decisions to have surgery are based on accurate perceptions

\section{Conclusion}

Women who chose to have surgery strongly believed that removal of breast tissue would significantly reduce their chances of developing the disease. The fact that such a high percentage of these women believed that they would inevitably develop cancer may explain both their decision to have the prophylactic surgery and their reduction in anxiety and psychological morbidity postoperatively.

We thank all the women in the study, who freely gave of their time, and all those who referred women to us, especially Gareth Evans, James Mackay, and Ros Eeles. We also thank Lida Graupner, who contributed to the early stages of the study, along with Kathryn Thirlaway and Angela Hall.

Contributors: LF, with Angela Hall and Kathryn Thirlaway, designed the pilot study. MBH was the primary coordinator of the study, conducting most of the interviews with women and collating and analysing the data. RA'H was the statistical adviser for the study from its inception. MBH, LF, and RA'H all contributed to final the analysis and writing of the paper. LF is the guarantor for the paper.

Funding: The study was funded by the Cancer Research Campaign. The pilot study was funded by a clinical research and development grant from the Middlesex Hospital Special Trustees Fund.

Competing interests: None declared.

1 Hartmann LC, Schaid DJ, Woods JE, Crotty TP, Myers JL, Arnold PG, et al. Efficacy of bilateral prophylactic mastectomy in women with a family history of breast cancer. N Engl J Med 1999;340:77-84

2 Zatz J. I'm definitely having it done. In: Marteau TM, Richards MPM, eds. The troubled helix: social and psychological implications of the new human genetics. Cambridge: Cambridge University Press, 1996:27-31.

3 Macke E. A family history of breast and ovarian cancer. In: Marteau TM, Richards MPM, eds. The troubled helix:social and psychological implications of the new human genetics. Cambridge: Cambridge University Press, 1996:31-7.

4 Lynch HT, Lynch J. Psychological aspects of monitoring high risk women for breast cancer. Cancer 1994;74:1184-92.

5 JW, Eeles R, Coles T, Taylor R, Lunt P, Baum M. Prophylactic mastectomy for genetic predisposition to breast cancer: the proband's [JW] story. Clin Oncol (R Coll Radiol) 1996;8:222-5.

6 Stefanek ME. Bilateral prophylactic mastectomy: issues and concerns. Natl Cancer Inst Monogr 1995; 17:37-42.

7 Borgen PI, Hill ADK, Tran KN, van Zee KJ, Massie M, Payne D, et al. Patient regrets after bilateral prophylactic mastectomy. Ann Surg Oncol 1998;5:603-6. 
8 Frost MH, Schaid DJ, Sellers TA, Slezak JM, Arnold PG, Woods JE, et al. Long-term satisfaction and psychological and social function following bilateral prophylactic mastectomy. JAMA 2000;284:319-24.

9 Lerman C, Croyle R. Psychological issues in genetic screening for breast cancer susceptibility. Arch Intern Med 1994;154:609-16.

10 Goldberg D, Williams P. A user's guide to the general health questionnaire. Windsor: NFER-Nelson, 1988.

11 Spielberger CD, Gorsuch RL, Lusherne R, Vagg PR, Jacobs GA. Manual for the state-trait anxiety inventory (form Y). Palo Alto, CA: Consulting Psychologists Press, 1983

12 Fallowfield L, Hall A, Maguire P. A question of choice: results of a prospective 3-year follow-up study of women with breast cancer. Breast 1994;3:202-8.

13 Thirlaway K, Fallowfield L, Cuzick J. The sexual activity questionnaire: a measure of women's sexual functioning. Qual Life Res 1996;5:81-90.

14 Fallowfield L. Psychological implication of tamoxifen treatment in women at high risk to breast cancer. In: Maltoni C, Soffritti M, Davis W, eds. The scientific bases of cancer chemoprevention. Amsterdam: Elsevier Science, 1996:219-25.

15 Stead ML, Crocombe WD, Fallowfield LJ, Selby P, Perren TJ, Garry R, et al. Sexual activity questionnaires in clinical trials: acceptability to patients with gynaecological disorder. Br J Obstet Gynaecol 1999;106:50-4

16 Folkman S, Lazarus RS. If it changes it must be a process: a study of emotion and coping during three stages of a college examination.J Persomality Social Psychol 1985;48:150-70.
17 Evans DGR, Blair V, Greenhalgh R, Hopwood P, Howell A. The impact of genetic counselling on risk perception in women with a family history of breast cancer. Br J Cancer 1994;70:934-8.

18 Hopwood P, Keeling F, Long A, Pool C, Evans G, Howell A. Psychological support needs for women at high genetic risk of breast cancer: some preliminary indicators. Psycho-oncology 1998;7:402-12.

19 Hopwood P, Fletcher I, Lee A, Al Ghazal S. A body image scale for use with cancer patients. Eur J Cancer (in press).

20 Al-Ghazal SK. The psychological impact of immediate rather than delayed breast reconstruction. Eur J Surg Oncol 2000;26:17-9.

21 Thirlaway K, Fallowfield L, Nunnerley H, Powles T. Anxiety in women "at risk" of developing breast cancer. Br J Cancer 1996;73:1422-4.

22 Watson M, Lloyd S, Davidson J, Meyer L, Eeles R, Ebbs S, et al. The impact of genetic counselling on risk perception and mental health in women with a family history of breast cancer. Br J Cancer 1999;79:868-74.

23 Thirlaway K, Fallowfield L, Evans G, Howell A. Entry into the tamoxifen prevention trial depends on women's estimates of the population risk of breast cancer. Breast 1995;4:203-4.

(Accepted 20 October 2000) 